\title{
Heat and power plant chimneys thermal inspection with an infrared camera
}

\author{
Katarzyna Stokowiec ${ }^{1 *}$, Dagmara Kotrys-Działak ${ }^{1},{\text { Dominika } \text { Mochocka }^{1} \text { and Marcin Sokołowski }}^{1}$ \\ ${ }^{1}$ Kielce University of Technology, Faculty of Environmental Engineering, Geomatics and Power Engineering, Kielce, Poland
}

\begin{abstract}
Thermography is getting more and more popular in many fields of everyday life nowadays. It is successfully applied in heat investigations. Infrared cameras imaging includes thermal analyses of building physics, thermal bridges or insulation improvement inspection. Flir E30 infrared camera was used in order to conduct measurements of two chimneys located in Kielce Power Station in Poland. The first tested facility is a structural steel chimney thermally isolated with mineral wool. The other chimney is built of reinforced concrete material. An infrared camera tests enabled the creation of the diagrams presenting the temperature distribution along the chimneys in different orientations. The research proved the correct functioning of both chimneys.
\end{abstract}

\section{Introduction}

Thermography, which can also be called infrared thermal imagining, is a distant thermal analysis method. This non-destructive way of assessing surface temperature of an examined object allows to determine the warmer and colder areas, which are presented using different colors on the image. The colors depend on the chosen palette in the technical software. In general, when the object presents warmer temperatures we can observe them by means of orange or red color with white one in the hottest areas. On the other hand, blue or green color mean the colder parts and black color appears at the spots with the lowest temperature range. The pictures are taken using an infrared camera (thermal imaging camera), which is a special device converting thermal image into color records. This method is based on observation by means of thermal imaging camera. The device records an infrared irradiance (IR) and converts it into visible light. The IR is sent by every body with the temperature higher than an absolute zero, what makes the technique available in wide range of experiments.

The term "thermovision" comes from the trade name assigned to the first thermovision camera produced by AGA. The change in body temperature was observed even around 400 years B.C. by Hippocrates, who analyzed the differences in the wet clay color during its desiccating on the skin. The quantity temperature measurements were also in the field of Galileo's interest. In 1800 Frederick William Herschel, a German astronomer and telescopes constructor, discovered infrared irradiance functioning during his research on energy transmitted by light colors.

The first infrared cameras were constructed and manufactured in the 70s of 20th century. Initially the technology was examined by the army in order to apply it in military actions. The infrared cameras were used during the $1^{\text {st }}$ World War as a device to localize the airplanes. The first THV cameras were massive and heavy but the technology development made them more practical and widely available.

The method is being currently applied in many fields of life. But still all of them involve heat transfer or heat treatment in wide range of applications $[1,2]$. One of them involves possible health problems examinations. The affected parts of the body present higher temperature than the rest and that is why we can observe the dysfunctions with an infrared camera. It involves not only human. Animals breeding sometimes require the early detection of health issues that may cause loss of money. The thermovision cameras are applied in order to detect the effects of the parity and stage of lactation on tissues changes of the dairy cows' hooves [3]. Not only animals but also people can be examined with usage of an infrared camera. The investigations e.g. include the temperature distribution deviations in oral cavity mucous membrane [4] in order to investigate changes under pathological conditions.

Moreover thermovision experiments involve laboratory simulations of several types of critical situations caused by human negligence or natural disasters. The results are to ensure optimum detection, warning, management and intervention at risk event [5]. The use of thermovision equipment enables a laboratory simulation of several types of aggravating circumstances such as sources of smoke, fog, rain, heat. Tests with infrared cameras can also easily facilitate the examinations of car engine's state [6], since the diagnostic computers not always clearly point the failure in components and its size. The infrared camera measurements of combustion engine elements

\footnotetext{
*Corresponding author: kstokowiec@ @u.kielce.pl
} 
as well as cooling system can be applied in optimal vehicle operation assessment. Car concerning research also includes heat exchangers [7]: their efficiency, possible phase-change heat exchangers with the use of microstructural coatings applications as well as heat transfer augmentation.

THV cameras are also applied by the fire department during rescue operation. In Poland infrared cameras documented the progress of extinguishing fire in manufacturing plant [8]. In such a case the temperature values are of highest importance. It is useful when deciding whether or not to finish fire extinguishing operation. Not only the external surface of the building on fire can be viewed by means of an infrared camera but also the internal area. While inside the manufacturing plant, the THV camera allowed [8] quick and efficient fire extinguish operation without additional damage to the construction: unnecessary holes in the walls, strop collapse or even excessive usage of extinguishing agents.

The research with an infrared camera allows the analysis of quality of joints obtained as a result of resistance welding of polypropylene pipes. Conducted studies showed the best results of joint during welding at a specific temperature range [9] of about $220^{\circ} \mathrm{C}$. The tests were performed for thermoplastic polymers connected with socket fusion welding not only with thermal image but also for flexural strength of the combined components. The received weld was also observed under a stereoscopic microscope. Other experimental measurements were conducted during multi-pass repair welding on crane wheels. They have been performed by thermovision camera and proved that the radiation component of heat dissipation and temperature dependence of material thermomechanical properties are essential factors [10] and the radiation effects should be included in order to improve the calculations.

Infrared thermography and building inspection is described as a new approach to teaching-learning process [11]. It is a new methodology to the physics of heat transfer and energy saving that deals with heat in building detected by means of an infrared camera. The thermography path is a multidisciplinary project to heat losses analyses. Students can study the surrounding world through physics and ask the practical questions they enquiry with their own observation. Therefore, on the basis of the images obtained using THV camera and the surface temperature distribution the optimal solutions for reducing energy losses can be established [12]. The measurements result in thermal bridges as well as defects recognition and therefore lowering the costs in terms of thermal energy usage. Then it is sufficient to examine only the thermal pattern. The heat losses analyses by means of thermography will improve energy efficiency and consequently influence the sustainable energy policy. They develop the thermal comfort inside the buildings for its users. The infrared thermovision can be easily applied as noninvasive investigation technique for the buildings constructed more than 20 years ago if it is difficult to find the design documents with the actual transmittances of the envelope [13]. Usually these buildings present massive constructions with high thermal inertia. The significant reduce in the heat transfer resistance of the shell may also be caused by defects of thermal protection through hinged facade systems used in buildings. An infrared camera was used in order to examine dependencies of the influence of the main defects on the increased heat transfer flow [14].

Thermal comfort is relevant for the indoor comfort especially in case of intelligent buildings. It can also be inspected by means of an infrared camera [15] and the suggested conclusions may enable the development of control algorithms for the building management system. That control optimal parameters provide maximal thermal comfort conditions for building occupants while minimizing the energy consumption of modern and intelligent buildings.

The thermography is also useful to identify areas at risk of mold growth in residential buildings. Thermal imaging camera can be used to create thermograms of building partitions [16], which enable determination of the possibility of mold appearance. The presence of moisture on thermograms on buildings surface visible as the difference in color is one of the preconditions for mold growth.

Historic buildings sometimes require thermal quality improvement. This task includes nondeterioration of their technical condition. Therefore the technical condition assessment must be evaluated using non-invasive methods, which means applying an infrared camera.

In case of building inspection with the exact determination of surface temperature the emissivity of material is of highest relevance. The inaccurate results may outcome in incorrect conclusions. Thus this parameter requires the laboratory tests, since it was proven that the values presented by the material manufacturer may differ from those estimated [17]. The tested quantities may also vary with moisture content. Thermography is mostly accepted as a reliable method to measure energy performance of existing buildings, in particular the thermal transmittance Uvalues of building envelope [18, 19]. Not only walls but also the windows may generate significant amount of heat loss. The results of thermographic analysis proved the accuracy of about $3.3 \%$ in case of $U$-value including glass and frame in situ for windows [20].

Infrared thermography not only involves building inspections or health issues. It concerns heat processes in general. That is why it can be applied by police forces in order to find fugitives. Recently in Poland thermovision cameras were employed in forests while searching for a criminal. Police also presents many achievements in locating elderly people, who left home and are highly probable to get lost, with THV cameras.

When taking measurements with an infrared camera we can observe a wide range of limitations [21] as well as errors of the method [22] that can be affected by many interferences while infrared radiation reaches the device. The errors can also be caused by the THV camera itself. 
Heat transfer and heat exchange involve not only infrared analysis. Heat exchangers require research concerning the tensile strength and therefore mechanical properties of porous structures consisting of copper wires reinforced with carbon and glass fibers were investigated. The tests allowed assessment of the adhesion strength of the porous structure produced as well as the cohesion between components of the structures [23].

The paper, as well as many presented publications, discusses the usage of infrared camera in order to proceed thermal analysis of the structure. The aim of thermal inspection research is to determine the proper work of two industrial constructions including the comparison of their functioning presented with temperature distribution along the chimneys.

\section{Methodology}

The experiments were conducted for two chimneys located in Kielce Power Station at Hubalczyków 30 in Kielce, Poland. These are two out of three existing chimneys.

Kielce Power Station (Polish: Elektrociepłownia Kielce) is a coal-fired, combined power and heat plant. It was established in 1987 and consists of one $140 \mathrm{MW}$ unit, six $25 \mathrm{MW}$ units and one $10 \mathrm{MW}$ cogeneration unit.

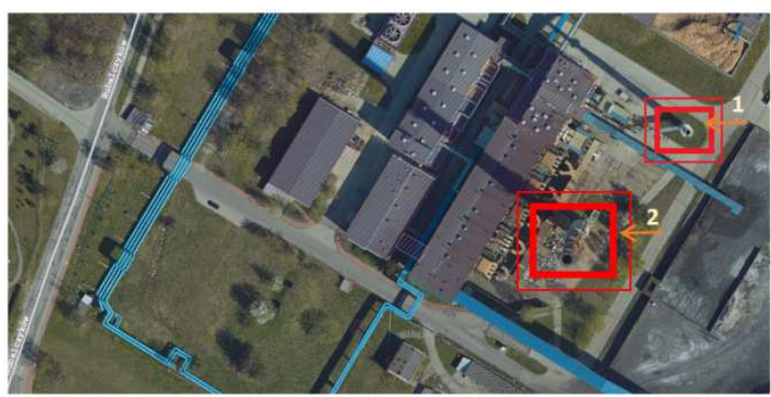

Fig. 1. Chimney location

1-steel chimney, 2-reinforced concrete chimney

Figure 1 presents the localization of both chimneys.

The first tested facility (1) was a structural steel chimney that is 80 meters high with the diameter at the base of 2,8 m. The chimney is thermally isolated with mineral wool of $10 \mathrm{~cm}$ thickness. The chimney emits exhaust gases produced during biomass combustion.

The experiments were also conducted for a reinforced concrete chimney (2). Its height is $120 \mathrm{~m}$ and the diameter at the base is $7,8 \mathrm{~m}$ with the wall thickness of $0,3 \mathrm{~m}$. This chimney is not thermally isolated. It emits the exhaust gases from coal dust combustion.

The temperature of the fuel gases at the outlet of both chimneys reaches the temperature of $140^{\circ} \mathrm{C}$.

The chimneys measurements took place on $8^{\text {th }}$ September 2020 at 12:00 - 1:00 p.m.. The experiments were conducted using two infrared cameras: Flir E30 and Flir S61. The measurements of ambient parameters (such as air temperature and relative humidity) as well as the distance between the device and the facility were carried out using Flir S61 camera, which was built in the smartphone.

However, the thermograms were taken by means of Flir E30 infrared camera. The camera presents the quality of the infrared image $160 \times 120$ pixels as well as temperature sensitivity below $0,1^{\circ} \mathrm{C}$. Flir E30 operates at a temperature range between -20 to $+350^{\circ} \mathrm{C}$ while the environment temperature range is between -15 to $+50^{\circ} \mathrm{C}$. The precision is $\pm 2{ }^{\circ} \mathrm{C}$ or $\pm 2 \%$ reading. We can see the thermal image choosing from 6 color palettes. While analyzing surface temperature we can also see the real image of the object by means of a 2 megapixels camera.

The structural steel chimney was measured in four different spots in four orientations while the reinforced concrete chimney only in three ones due to the conservation works.

\section{Results}

An infrared camera registration allows the observation of the thermal image on the outer surface of the analyzed chimney. We can also receive the digital photo of the existing object.

In case of steel chimney, the pictures were taken at the bottom and at the top of the facility, whereas the reinforced concrete chimney was pictured only once in each direction.

Among the parameters required to obtain technically proper thermogram the most important is emissivity coefficient of the experimented object material. When entered to the infrared camera software it should have the proper value in order not to cause errors in results. Therefore, the materials emissivity values were included during the thermal imaging camera tests.

a.)

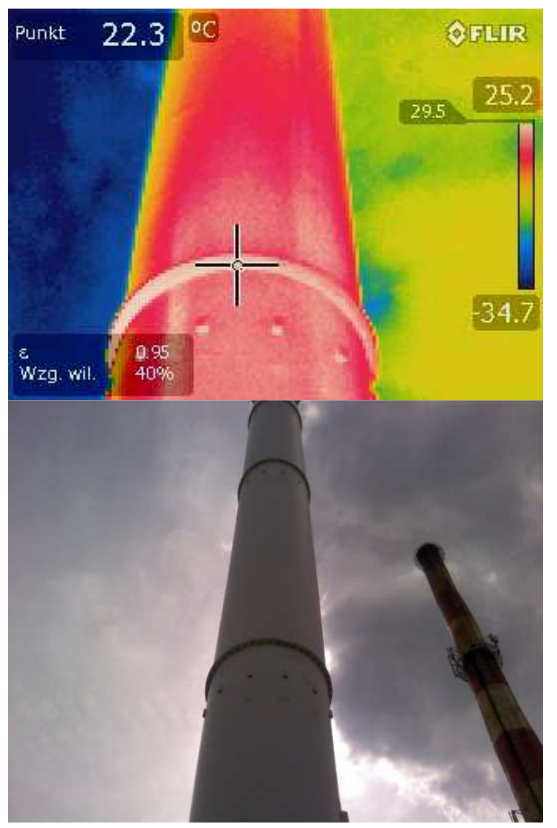

Fig. 2. Structural steel chimney picture - top of the facility (north side) a.) thermal image, b.) digital photo 
a.)

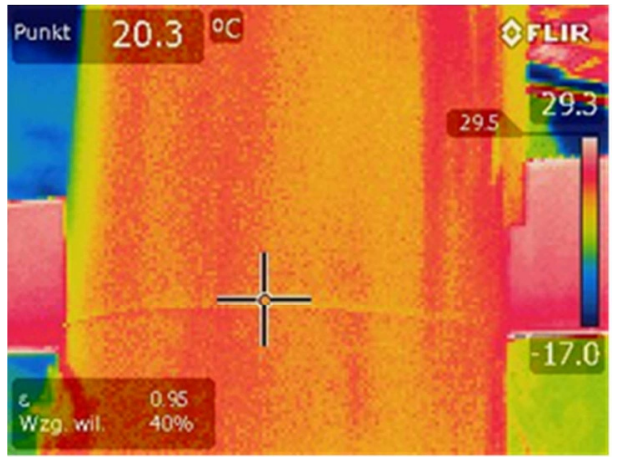

b.)

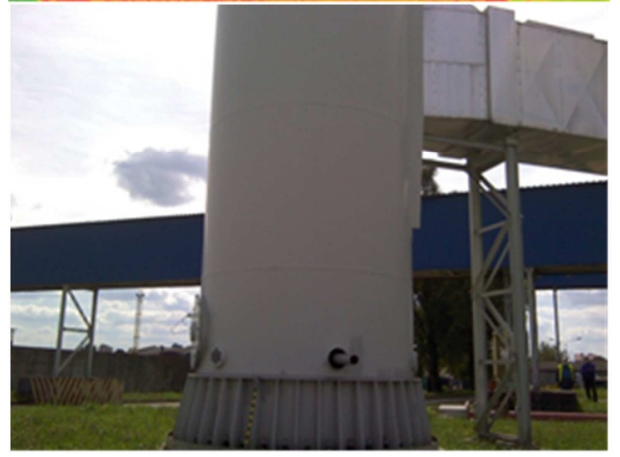

Fig. 3. Structural steel chimney picture - bottom of the facility (north side) a.) thermal image, b.) digital photo

a.)

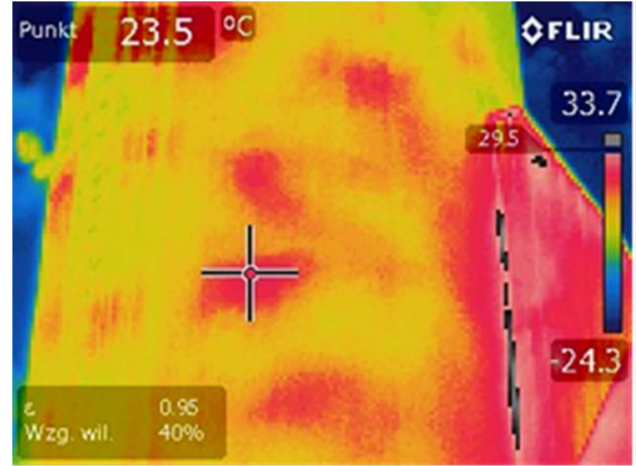

b.)

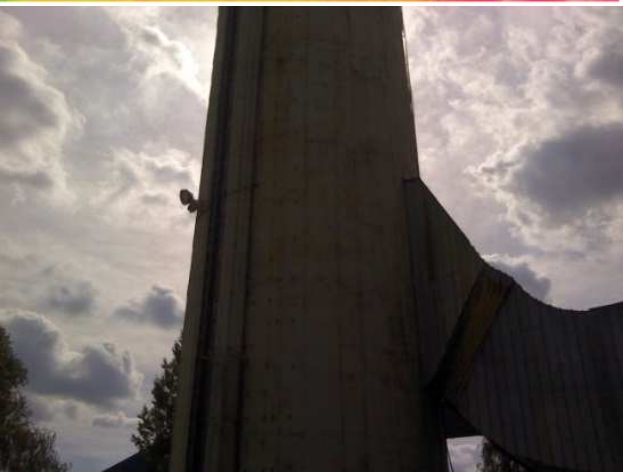

Fig. 4. Reinforced concrete chimney picture (north side) a.) thermal image, b.) digital photo

As we can observe the thermograms for structural steel chimney are more homogenous, while the reinforced concrete image presents several spots shown as darker parts on image that may indicate some cracks or leakiness, since they present areas of higher temperature. Therefore, even the infrared pictures itself could be one of the easiest and the most efficient ways to conclude whether the construction has tight walls. The first assumptions may indicate the necessary conservation works or on the other hand when the thermal image presents no possible damage the facility owner may avoid unnecessary heavy works.

But still the thermal analysis on the basis of an infrared pictures should be conducted.

It is necessary to enter into consideration the limitations of infrared cameras applications. Before conducting the measurements or during the data proceeding many information is required to be involved. The parameters introduced into the device software include: emissivity, the distance between the analyzed object and the thermal imaging camera, the ambient temperature as well as the humidity. All of the above conditions were fulfilled during the described research.

The parameters such as the distance to the structure, ambient temperature and humidity were measured by means of Flir S61 camera.

The emissivity was introduced to the Flir E30 infrared camera device software. The values were assumed for each chimney separately basing on its building material on the external surface: mineral wool or reinforced concrete ( 0.85 and 0.95 respectively).

\section{Analysis}

An infrared camera measurements enabled the creation of the diagrams of temperature distribution along the chimneys in different orientations.

Figure 5. presents the temperature distribution in the horizontal direction on both steel and reinforced concrete chimney. The analyses were selected for east and north orientation since the research was conducted in this orientation for both chimneys due to the conservation works and problems with taking thermal images of reinforced concrete chimney.

The straight line shown in Fig. 5. was chosen for a chimney made of structural steel, while the dashed one for the one built with reinforced concrete.

As it is shown in east direction the temperatures decline along each chimney whereas the diagrams for north orientation present the opposite tendency: the temperatures mostly increase. The temperature difference in each case is low (up to 6K). The differences in temperature in each direction are similar no matter the chimney material.

The east orientation presents higher temperatures along the line for both structural steel as well as reinforced concrete chimney than the north orientation. The outer surface temperature of the facilities increases faster due to the greater solar operation on the east side than on the north one.

Steel and reinforced concrete present dissimilar heat conduction values. Therefore we can observe various temperature distribution on each side of both chimneys. 
a.)

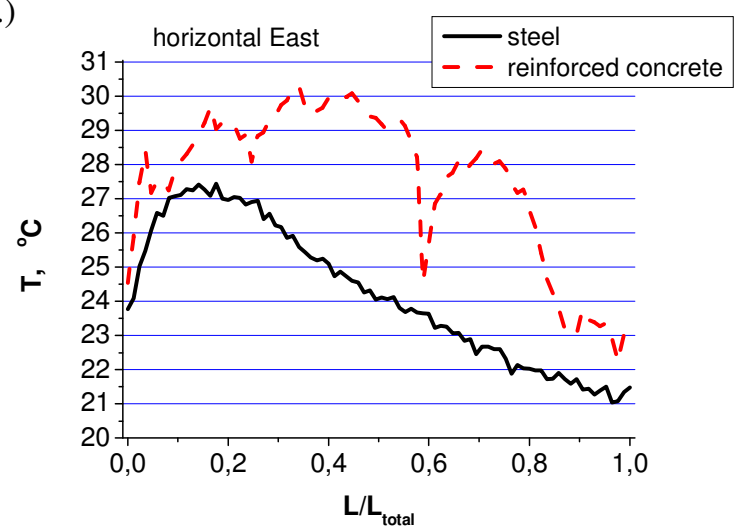

b.)

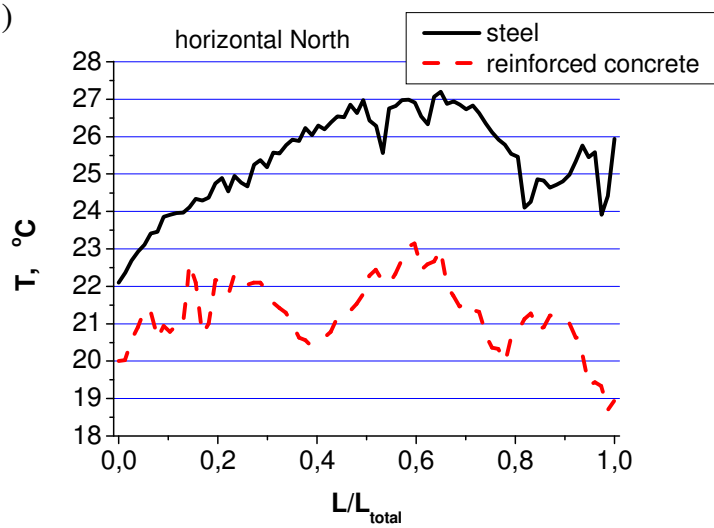

Fig. 5. Temperature distribution along the chimneys in horizontal direction oriented: a.) East, b.) North

The diagrams present many sudden temperature drops, which is more visible in case of the chimney made of reinforced concrete. The declines in temperature prove numerous discontinuities on the outer surface of the chimney.

What is more, we observe higher temperatures along the structure on the reinforced concrete chimney in east direction, whereas the opposite tendency is presented in north direction. From the curves it may be assumed that the reinforced concrete chimney preheats faster but the structural steel one conveys less heat to the ambient.

Figure 6. pictures the temperature distribution only along the structural steel chimney in horizontal direction. The lines present the bottom (straight one) and the top (dashed one) of the chimney. The measurements were performed by means of thermal imaging camera taking two photos along this chimney in each orientation $(\mathrm{N}, \mathrm{S}, \mathrm{E}, \mathrm{W})$ but only south and west are samples shown in Figure 6.

The curves mainly present the lower temperatures at the bottom of the chimney. The combustion gases decrease their temperature during the flow to the chimney outlet. Moreover they are subject of the wind operation. Therefore the higher they reach, the temperature decline is more visible.

The south orientation presents higher temperatures values along the horizontal line for both bottom and top of the structural steel chimney than the west orientation. The reason for that phenomenon is the sun's migration on the horizon during the day. Solar operation faster reaches the chimney walls on the south side than on the west one.

In Figure 5. and 6. we can also observe several sudden temperature drops which can be explained by means of several cracks and/or joints on the chimneys' surface.

a.)

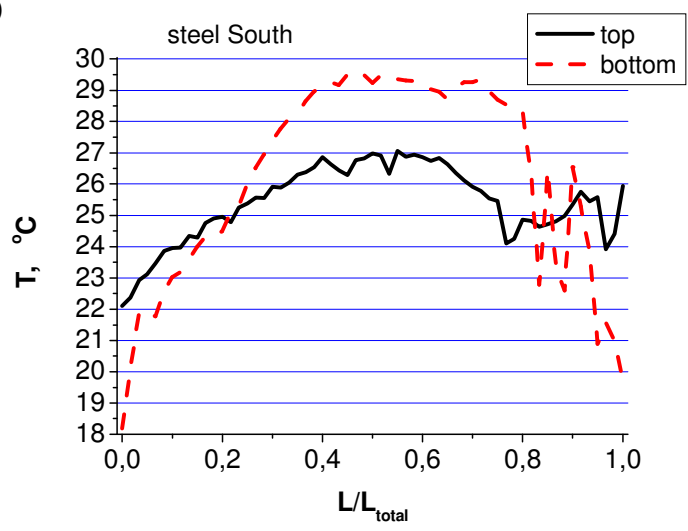

b.)

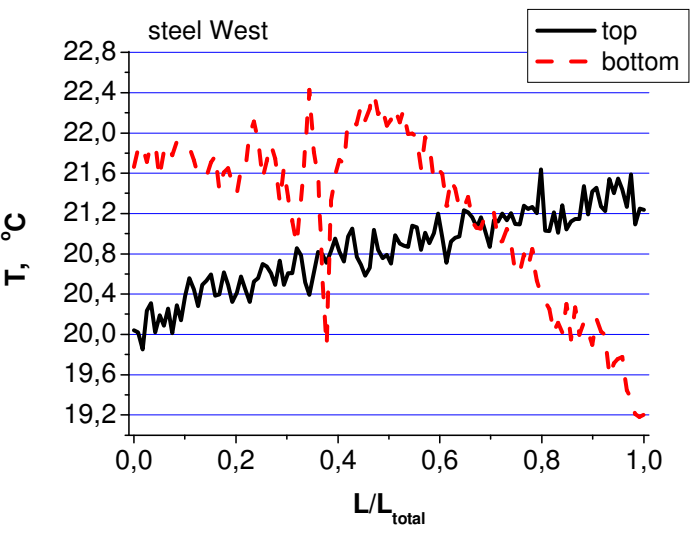

Fig. 6. Temperature distribution along the structural steel chimney at the bottom and top of it in horizontal direction oriented: a.) South, b.) West

In Figure 5 and 6 in most cases the edge temperatures present lower values. Since the lines on the thermogram drawn in order to prepare the curves were in horizontal direction, such temperature distribution is obvious. Both chimney have cylindrical shape, and therefore the results located in the middle of the length (ca. 40-60\%) are the most reliable. The temperatures at both margins are subject to the radiation process.

While analyzing the diagrams we observe that the temperature distribution along the chimney presents the characteristics akin to quadratic function.

While studying the temperature distribution along the structural steel chimney at the bottom and top of it in horizontal direction (Fig. 5) the dependences were prepared. As it was mentioned, the quadratic function was employed in order to fit the average temperature values along the structure in each direction: 


$$
T=\mathrm{A}+\mathrm{B}_{1} \cdot \frac{L}{L_{t}}+\mathrm{B}_{2} \cdot\left(\frac{L}{L_{t}}\right)^{2}
$$

$\mathrm{T}$ - temperature, ${ }^{\circ} \mathrm{C}, \mathrm{L}$ - the edge distance, $\mathrm{m}, \mathrm{L}_{\mathrm{t}}-$ total length, $\mathrm{m}, \mathrm{A}, \mathrm{B}_{1}, \mathrm{~B}_{2}-$ constants

The constants depend on the orientation:

Table 1. The quadratic function constants.

\begin{tabular}{|c|c|c|}
\hline Constant & South & West \\
\hline A & 20,77 & 20,76 \\
\hline B1 & 27,39 & 2,54 \\
\hline B2 & $-25,73$ & $-2,99$ \\
\hline
\end{tabular}

The fit with quadratic function presents high values of coefficient of determination: 0.95 for south orientation and 0.66 for west orientation. The values prove that $95 \%$ of outcomes are replicated by the model in case of south side of the chimney and $66 \%$ in case of west side.

The other statistical rate that was calculated was standard deviation. Its values in south and west orientation are 0.5 and 0.19 , respectively, which indicates that the values tend to be close to the mean of the set (also called the expected value).

\section{Conclusions}

On the basis of taken measurements as well as the proposed analysis presented on diagrams we can observe few dependences.

The visible sudden decrease or increase in temperature are located on the construction joints of the chimneys.

The thermal images results are strongly dependent on atmospheric conditions. The thermograms on the sun-filled side present higher temperatures. It is necessary to take this variable into consideration during the analysis, since the temperature difference not necessary proves the massive heat flow but may only mean the higher solar radiation.

There are other factors influencing the given effects such as measurement conditions, precision of used infrared camera, heat penetrability or emissivity of the material.

Figure 5. presents temperature distribution along both chimneys in horizontal direction. The discrepancies in observed temperatures arise from the differences in the chimneys' structure: the material, its thermal conductivity, whether or not there is an isolation.

The diagrams for steel chimney (Figure 6) bottom and top thermal images prove the temperature differences between those two parts are negligible. Still the top of the chimney presents other temperatures due to the wind exposure. On the South side of the steel chimney (Figure 6a) we observe the temperature drop at the edge of the chimney that can be explained by the radiation impact. The most reliable temperature results are in the middle of the chimney which has cylindrical shape.

The diagrams present the temperature distribution along the chimney akin to quadratic function. The calculations prove that such characteristic is approximate. The statistics implicate high values of coefficient of determination and low values of standard deviation.

The experiments demonstrate that the infrared camera is a significant measurement tool in case of chimney analysis. Both tested chimneys: steel and reinforced concrete, are efficient. The structural steel one presents to have a sufficient and functional isolation, whereas the reinforced concrete one works properly even without the isolation.

\section{References}

[1] S. Wciślik, J. Phys. Conf. Ser., 745, 032064 (2016) [2] T. Orzechowski, S. Wciślik, Int. J. Heat Mass Transf., 73, 110-117 (2014)

[3] T. Bobici, P. Mijic, V. Gantner, H. Glavas, M. Gregic, J. Cent. Eur. Agric., 19(4), 777-783 (2018)

[4] M. Dazbrowski, R. Dulski, S. Zmuda, P. Zaborowski, C. Pogorzelski, Infrared Phys. Technol., 43, 265-269 (2002)

[5] C. Spulber, O. Borcan, A. Bobei Sterian, C. Sarafoleanu, P. Sterian, Environ Eng Manag J, 18(5), 1067-1076 (2019)

[6] A. Nieoczym, TTS 10, 1951-1958 (2013)

[7] Ł. J. Orman, R. Chatys, Heat transfer augmentation possibility for vehicle heat exchangers, Proceedings of International Conference "Transport Means", Lithuania, 9-12 (2011)

[8] Ł. Łacioki, J. Rybiński, A. Szajewska, Technika I Technologia BiTP, 30, 75-80 (2013)

[9] A. Kalwik, K. Mordal, J. Redutko, Arch. Metall. Mater. 66 2, 511-518 (2021)

[10]A. S. Sedmak, D. R. Tanaskovi, A. C. Murariu, Therm. Sci., 21 (2), 1003-1009 (2017)

[11]M. Carpineti, L. Cazzaniga, L. Perotti, M. Giliberti, M. Cavinato, N. Ludwig, Can. J. Phys. 97, 1019-1026 (2019)

[12]A. Preda, L. L. Popescu, S. R. Popescu:. Revista Română de Inginerie Civilă, 10(4), 426-433 (2019)

[13]R. Pescaru, L. Dumitrescu, I. Baran, D. Isopescu, Application of the infrared thermography for the evaluation of energy conservation in built-up local communities, 17th International Multidisciplinary Scientific GeoConference SGEM, 17-25 (2017)

[14]A.E. Rusanov, A.Kh. Baiburina, D.A. Baiburin, V. Bianco, Mag. Civ. Eng., 95(3), 57-65 (2020)

[15]E. Barreira, R. M. S. F. Almeida, M. L. Simões, Sens., 21, 1961 (2021)

[16]R. Stachniewicz, Ekonomia i Środowisko, 3 (66), 142-156 (2018)

[17]M. Fedorczak-Cisak, E. Radziszewska-Zielina, B. Orlik-Kożdoń, T. Steidl, T. Tatara, Energies, 13, 4610 (2020) 
[18]Rossano Albatici, Arnaldo M. Tonelli: Energy Build, 42, 2177-2183 (2010)

[19]V. Tzifa, G. Papadakos, A. G. Papadopoulou, Vaggelis Marinakis, J. Psarras, Int. J. Sustain. Energy, 36 (1), 28-46 (2017)

[20]S. Park, S. H. Kim, H. Jeong, S. L. Do, J. Kim, Energies, 14, 1904 (2021)

[21] T. Orzechowski, COW, 43 (10), 446-448 (2012)

[22]K. Stokowiec, Instalacje solarne $\mathrm{z}$ akumulacją ciepła. Politechnika Świętokrzyska, 2021

[23] R. Chatys, Ł. J. Orman, Mech. Compos. Mater., 53, 351-360 (2017) 